\title{
A HIPERINSUFLAÇÃO PULMONAR INDUZIDA PELO VENTILADOR MECÂNICO EM PACIENTE INTUBADOS COMO TERAPIA DE HIGIÊNE BRÔNQUICA
}

\author{
Marconi José Chaves*, Maria José Ramalho Pedreira**, José Edmilton Felix***, Carlos Alcino Filho****, \\ Abrahão Fontes Baptista*****

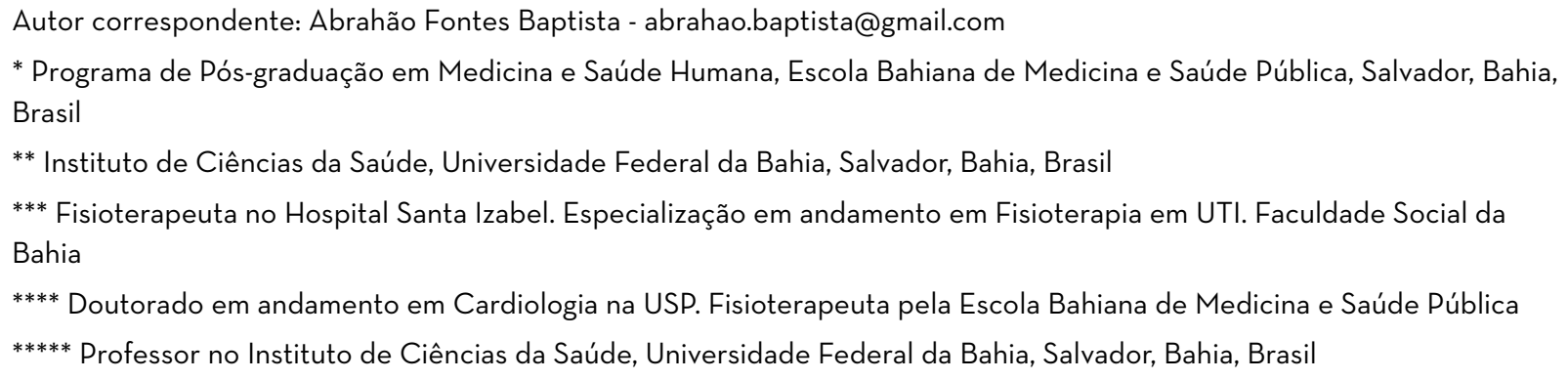

\begin{abstract}
Resumo
Introdução: A fisioterapia respiratória na unidade de terapia intensiva (UTI) visa promover independência respiratória funcional, prevenir complicações e acelerar a recuperação. O objetivo foi verificar o efeito da hiperinsuflacao pulmonar utilizando o ventilador mecânico, na mecânica do sistema respiratório e hemodinâmica. Materiais e Métodos: Este estudo envolve um ensaio clínico aberto onde foram incluídos 18 pacientes em ventilação mecânica. Foram avaliados os parâmetros da mecânica pulmonar, hemodinâmica, saturação de oxigênio, volume corrente inspirado e o pico de fluxo expiratório. Resultados: Não foram evidenciadas alterações nos parâmetros da mecânica do sistema respiratório e hemodinâmica, porém os valores de pico de fluxo expiratório e o volume corrente inspirado aumentaram significativamente durante a técnica $(\mathrm{P}<0,05)$. Conclusões: $\mathrm{A}$ hiperinsuflação pulmonar com o ventilador mecânico parece ser segura, pois não promoveu impacto clínico em variáveis hemodinâmicas e pode ser utilizada como coadjuvante na terapia de higiene brônquica devido ao seu potencial em aumentar o pico de fluxo expiratório.
\end{abstract}

Palavras-chave: Terapia de higiene brônquica; Ventilação mecânica; Hiperinsuflação pulmonar. 


\title{
LUNG INSUFFLATION BY ARTIFICIAL RESPIRATION AS AIRWAYS HIGYENE
}

\begin{abstract}
Introduction: Respiratory physical therapy in intensive care units (ICUs) aims to promote functional respiratory independence to the patient, preventing complications and accelerating recovery. The objective of the study was to assess the effects of lung hyper insufflation through artificial respiration in the respiratory system and in hemodynamics. Methods: a self controlled clinical trial on a sample of 18 patients on artificial respiration. Pulmonary mechanics, hemodynamics, oxygen saturation levels, current inspired volume and expiratory flux peak parameters were assessed and evaluated. Results: No evidence was found regarding hemodynamics and respiratory system mechanics but expiratory flux peak values increased significantly $(P<0.05)$. Conclusion: lung insufflation through artificial respiration appears to be safe because it has caused no clinical impact em hemodynamics e may be employed as additional therapy to clean airways due to its potential to increase expiratory flux peak.
\end{abstract}

Keywords: Artificial Respiration; Insufflation; Cough.

\section{INTRODUÇÃO}

Pacientes internados em Unidade de Terapia Intensiva (UTI's), com via aérea artificial, em ventilação mecânica, são candidatos a desenvolverem infecções respiratórias, devido a alterações no clearence mucociliar, promovendo aumento na produção de secreção. Os anestésicos freqüentemente usados nestas condições também levam a hipoventilação e a hipoxemia, alterando a complacência pulmonar, inibindo assim o mecanismo fisiológico da tosse. ${ }^{(1,2)}$

Estes pacientes apresentam necessidades especiais, as quais, na maioria das vezes exigem assistência sistematizada, além de uma série de cuidados, objetivando evitar complicações. As manobras fisioterapêuticas relacionadas aos cuidados respiratórios consistem em técnicas manuais, posturais, instrumentais ou que utilizem recursos do ventilador, a fim de garantir higienização e a ventilação pulmonar. ${ }^{(3,4)}$
Uma tosse ineficaz levará a retenção de secreções, consequentemente a hipoventilação alveolar. $(6,7)$ Características como a força dos músculos expiratórios, pico de fluxo de tosse e o volume corrente pulmonar (VC), são determinantes da sua efetividade. Estima-se que uma inspiração entre 60 a $80 \%$ da capacidade pulmonar total está correlacionada com o deslocamento efetivo de secreções nas vias aéreas. ${ }^{(8)}$ Quando o paciente está ventilado mecanicamente de forma invasiva, é possível controlar o volume corrente inspirado administrado e promover seu incremento com o objetivo de auxiliar de forma coadjuvante na eliminação de secreções.

Em 1868, Hering e Breuer observaram o papel do nervo vago no controle da respiração e relataram à existência do reflexo que levou os seus nomes e tem função de diminuir a atividade muscular inspiratória com elevados VC prevenindo assim a hiperdistensão dos alvéolos e os volutraumas. ${ }^{(12-14)}$ Apoiado neste princípio, hipotetizamos que o uso 
de volumes correntes mais altos do que os preconizados atualmente podem ser usados para provocar uma tosse mais efetiva em pacientes ventilados mecanicamente, sem induzir o risco de lesões volumétricas ou alterações hemodinâmicas. Neste sentido, este trabalho teve como objetivo verificar o efeito da hiperinsuflação pulmonar utilizando o ventilador mecânico, na mecânica do sistema respiratório e hemodinâmica.

\section{MATERIAIS E MÉTODOS}

O presente estudo trata-se de um ensaio clínico aberto, realizado no período de julho a setembro de 2009 na UTI clínica de um hospital privado na cidade de Salvador, Brasil. O projeto foi realizado após a aprovação pelo Comitê de Ética em Pesquisa do Hospital Santa Izabel de acordo com a resolução 196/96 do Conselho Nacional de Saúde.

\section{SELEÇÃO DOS PACIENTES}

Foram incluídos neste estudo pacientes em ventilação mecânica controlada, que estivessem entubados ou traqueostomizados e sedados. Foram excluídos os pacientes que apresentaram as seguintes condições clinicas: instabilidade hemodinâmica ou cardíaca, pós-operatório de cirurgias com risco de rompimento da anastomose, histórico de vômitos consecutivos, hemorragia alveolar ou presença de fragilidade vascular, fistula bronco-pleural, pneumotórax, tórax instável, crise de hiperatividade brônquica, edema agudo de pulmão cardiogênico, traumatismo crânio encefálico (TCE) com monitorização da Pressão Intracraniana (PIC) estando com Derivação Ventricular Externa (DVE) fechada, fibrose pulmonar, SARA, lesão medular em $\mathrm{C}_{1}, \mathrm{C}_{2}, \mathrm{C}_{3}$ ou curarização.

\section{PARÂMETROS AVALIADOS}

Os parâmetros avaliados antes, durante e após a intervenção foram a pressão de pico (Ppico), pres- são de platô (Pplatô), complacência estática (Cest), pressão resistiva (Presistiva), volume corrente inspirado, pico de fluxo expiratório (PFE), saturação periférica de oxigênio ( $\left.\mathrm{SpO}_{2}\right)$, frequência cardíaca (FC) e pressão arterial media (PAM).

A saturação periférica de oxigênio foi avaliada através de um oxímetro de pulso (Dixtal 2010, Alemanha), colocado no quarto dedo da mão. O comportamento da hemodinâmica foi avaliado através dos valores de frequência cardíaca e a pressão arterial média que foram registrados através de um monitor de sinais vitais (Dixtal 2010, Alemanha).

Para a medida da pressão de pico (Ppico) e de platô (Pplatô) do sistema respiratório foi considerado o valor demonstrado no visor do ventilador mecânico, medido em $\mathrm{cmH}_{2} \mathrm{O}$. A pressão de platô foi obtida através da técnica de oclusão rápida das vias aéreas durante uma insuflação com fluxo constante (onda quadrada) com pausa inspiratória de 4 segundos. A complacência estática (Cest) foi calculada a partir da pressão de platô (Pplatô), da pressão positiva expiratória final (PEEP) e do volume corrente $(\mathrm{Vc})$ sendo obtida através da formula:

$$
\text { Cest }=\text { Vt / Pplatô }- \text { PEEP }
$$

A pressão resistiva (Presistiva) foi obtida pela fórmula:

$$
\text { Pres }=\text { Pressão de pico }(\text { Ppico })-\text { Pplatô }
$$

\section{INTERVENÇÃO}

Todos os pacientes estavam ventilados no NELLCOR PURITAN BENNETT 840 VENTILATOR SYSTEM, modo volume controlado (VCV), posicionados em decúbito dorsal, com a cabeceira do leito a $35^{\circ}$ graus de inclinação. Os ajustes ventilatórios iniciavam com o alargamento do limite de pressão para $100 \mathrm{cmH}_{2} \mathrm{O}$, mantendo o PEEP fisiológico (5 $\mathrm{cmH}_{2} \mathrm{O}$ ), frequência respiratória de 8 incursões por minuto, taxa de fluxo de $40 \mathrm{~L} / \mathrm{min}$, utilizando onda descendente e o volume corrente foi elevado para 
2 L. Eram realizados então oito ciclos respiratórios, sendo que a técnica poderia ser interrompida se houvesse sinais de instabilidade hemodinâmica, caracterizada por uma PAM inferior a $60 \mathrm{mmHg}$, bradicardia, ou $20 \%$ da queda da frequência cardíaca basal.

Após aplicação da técnica foi realizada a segunda monitorização, feita sem a realização de nenhuma intervenção e posteriormente foi realizada aspiração traqueal, seguindo o protocolo de aspiração descrito por Irwin et al. 2003 e utilizando uma sonda de aspiração traqueal $n^{\circ} 14$.

\section{ANÁLISE ESTATÍSTICA}

Para descrição das variáveis contínuas foram utilizadas medidas tendência central e dispersão. Para verificar a existência de diferenças significativas entre os momentos avaliados usamos o teste Exato de McNemar (usando distribuição binomial) entre as variáveis dicotômicas, nas variáveis continuas o teste de Exato de Wilcoxon para amostras emparelhadas quando comparamos dois momentos $e$ o Exato de Friedman seguidos do teste a posteriori de Dunn quando comparamos os três momentos. Para comparar a PFE antes do procedimento com o valor atingido durante o procedimento usamos o teste Exato de Mann-Whitney Foram consideradas como variáveis dependentes os parâmetros respiratórios e hemodinâmicos e como variáveis independentes o uso da técnica de hiperinsuflação. $\mathrm{O}$ valor de alfa foi estabelecido em $5 \%(p<0,05)$.

\section{RESULTADOS}

Foram incluídos no estudo 18 pacientes, sendo 12 do sexo feminino, com mediana da idade de 70 anos ( $Q_{1}$ 59,25 e $\left.Q_{3} 80,00\right)$ com altura 1,60 m ( $Q_{11,58} \mathrm{~m} \mathrm{e}$ $\left.\mathrm{Q}_{3} 1,72\right) \mathrm{m}$ e peso $57 \mathrm{Kg}\left(\mathrm{Q}_{1} 50 \mathrm{Kg}\right.$ e $\left.\mathrm{Q}_{3} 65 \mathrm{Kg}\right)$, características evidenciadas na tabela 1.

Tabela 1 - Característica da amostra

\begin{tabular}{|c|c|}
\hline CARACTERÍSTICAS & Mediana (Q1 - Q3) \\
\hline IDADE (ANOS) & $70(50,25-80)$ \\
\hline PESO (KG) & $57(50-65)$ \\
\hline ALTURA (M) & $1,60(1,58-1,72)$ \\
\hline
\end{tabular}

Os parâmetros da mecânica do sistema respiratório Cest, Ppico, Pplatô e Presistiva não se modificaram após a hiperinsuflação pulmonar em relação aos valores basais (tabela 2). Comportamento semelhante foi observado nos parâmetros hemodinâmicos FC e PAM (tabela 3).

Tabela 2 - Comportamento da mecânica do sistema respiratório antes e após a hiperinsuflação pulmonar

\begin{tabular}{cccc}
\hline & ANTES & APós & P VALOR \\
\hline Ppico $\left(\mathrm{cmH}_{2} \mathrm{O}\right)$ & $27,5 \mathrm{O}(23,5 \mathrm{O}-31)$ & $25,5 \mathrm{O}(19-29,25)$ & $<0,257$ \\
Pplatô $\left(\mathrm{cmH}_{2} \mathrm{O}\right)$ & $20,5 \mathrm{O}(16,75-24,25)$ & $19,5 \mathrm{O}(15-25)$ & $<\mathrm{O}, 309$ \\
Presistiva $\left(\mathrm{cmH}_{2} \mathrm{O}\right)$ & $6,5 \mathrm{O}(2,75-13)$ & $4(2-8,25)$ & $<0,423$ \\
Cest $\left(\mathrm{ml} / \mathrm{cmH}_{2} \mathrm{O}\right)$ & $39,5 \mathrm{O}(27,5 \mathrm{O}-45,25)$ & $43,5 \mathrm{O}(3 \mathrm{O}-47)$ & $<0,111$ \\
\hline
\end{tabular}

Valores expressos em termos de mediana (Q1-Q3) Ppico - Pressão de pico, Pplatô - Pressão de platô, Presistiva - Pressão resistiva, Cest - Complacência estática 
Tabela 3 - Comportamento da hemodinâmica antes, durante e após a hiperinsuflação pulmonar

\begin{tabular}{ccccc}
\hline & ANTES & DURANTE & APÓs & P VAlOR \\
\hline FC $(\mathrm{mmHg})$ & $88(69,75-110)$ & $81(71,25-93,25)$ & $85,50(74,75-105,25)$ & $<0,1225$ \\
PAM $(\mathrm{mmHg})$ & $82(75,75-102,25)$ & $83(66-92)$ & $83,50(73,75-98,25)$ & $<0,0919$ \\
\hline
\end{tabular}

Valores expressos em termos de mediana (Q1-Q3) FC - Freqüência cardíaca, PAM - Pressão arterial média, SpO2 - Saturação periférica de oxigênio

A intervenção levou a um aumento de mais do que três vezes no volume corrente durante o período de hiperinsuflação, retornando após este período a valores basais ( $p<0,0001)$ (tabela 4$)$. O mesmo aconteceu com o PFE, que apresentou aumento de qua- tro vezes em relação aos valores basais $(\mathrm{p}<0,0001)$ (Tabela 4). A SpO subiu de $96 \%\left(Q_{1}\right.$ 94,75\% e Q $97 \%)$ antes da intervenção, para $97 \%\left(Q_{1} 96,75 \%\right.$ e $\mathrm{Q}_{3} 98 \%$ ) durante e $98 \%$ (Q $97 \%$ e $\mathrm{Q}_{3} 99 \%$ ) após $(\mathrm{p}<0,0001)$.

Tabela 4 - Comportamento do volume corrente inspirado e pico fluxo expiração antes e durante a hiperinsuflação pulmonar

\begin{tabular}{cccc}
\hline & ANTES & DURANTE & P VALOR \\
\hline Vt insp $(\mathrm{ml})$ & $500(425,75-530,75)^{*}$ & $1.674,50(1.551,50-1.856,25)^{*}$ & $<0,0001^{*}$ \\
PFE $(\mathrm{l} / \mathrm{min})$ & $50(40-60)^{*}$ & $200^{*}$ & $<0,0001^{*}$ \\
\hline
\end{tabular}

Valores expressos em termos de mediana (Q1-Q3) Vt inspi - Volume corrente inspirado, PFE - Pico de fluxo expiratório.

\section{DISCUSSÃO}

Este estudo partiu do princípio de que a presença do Reflexo de Hering-Breuer permitiria um uso momentâneo de volumes correntes maiores do que os preconizados na literatura, o que poderia gerar um pico de fluxo expiratório mais efetivo para a remoção de secreções na via aérea de pacientes entubados e ventilados mecanicamente. Os resultados mostram que a hiperinsuflação pulmonar provocada com o ventilador mecânico aumentou o fluxo expiratório sem modificar parâmetros hemodinâmicos ou da mecânica do sistema respiratório de pacientes internados em UTI.

A literatura se mostra bastante divergente a respeito do volume corrente necessário para a ativação do Reflexo de Hering-Breuer. ${ }^{(3,17,18)}$ Os valores comumente descritos variam de $800 \mathrm{ml} \mathrm{a} 1500 \mathrm{ml}$, para desencadear esse reflexo em adultos eupnéicos conscientes. O limita a inspiração, evitando lesões por barotrauma. Por outro lado, volumes correntes maiores podem levar a uma tosse mais efetiva. Neste estudo verificamos que a hiperinsuflação com valores em torno de $1500 \mathrm{ml}$ levaram à tosse, expressa pela aumento no pico de fluxo expiratório.

O deslocamento de secreções promove alterações no componente resistivo que podem ser evidenciados pela modificação de variáveis da mecânica como a Resistência do sistema respiratório, Presistiva e Ppico. ${ }^{(6,7)}$ Porém nosso estudo essas alterações não apresentaram variações estatisticamente significativas. A Cest do sistema respiratório não se modificou durante todos os momentos da avaliação.

O uso de altos valores de volume corrente poderia ter influenciado em parâmetros hemodinâmicos como a FC e PAM, o que comprometeria o uso da intervenção. $O$ volume corrente excessivo poderia também impedir o retorno venoso e dimi- 
nuir a pré-carga ventricular, diminuindo debito cardíaco e ainda ativando o sistema parassimpático por resposta vagal, o qual poderia ocasionar uma bradicardia reflexa. ${ }^{(11,12)}$ Entretanto, nossos resultados não mostraram tais alterações. Esse comportamento pode ser atribuído ao aumento do volume corrente e a ativação do reflexo de Hering-Breuer, que reduz a sensibilidade do quimiorreceptor, poderia realçar a eficiência do barorreceptor e reduzir a pressão arterial e a ativação simpática. ${ }^{(10)}$

Em relação ao PFE foi evidenciado um incremento significativo, atingindo valores de $200 \mathrm{l} / \mathrm{min}$. Estudos consideram que pacientes com PFE menores de $160 \mathrm{l} / \mathrm{min}$ não possuem fluxo adequado para remoção de secreções e necessitam de auxilio para viabilizar esta função. ${ }^{(13,14)} \mathrm{A}$ avaliação da $\mathrm{SpO}_{2}$ mostrou um aumento após a hiperinsuflação com o ventilador mecânico e aspiração traqueal. Pode-se considerar que essa melhora seja devido o aumento da área disponível para trocas gasosas após o deslocamento e remoção de secreção.

Um estudo relatando o comportamento da secreção submetida a diferentes fluxos e situações clínicas levantam a hipótese que a eficácia de técnicas que visam a higiene brônquica necessitam de um pico de fluxo expiratório maior que o pico de fluxo inspiratório, pois inversamente a secreção tenderia a ser conduzida para regiões mais distais. ${ }^{(2)}$

O aumento do PFE encontrado no estudo pode estar associado com o incremento de volume corrente promovendo como consequência o aumento da pressão de recolhimento elástico durante a expiração. Resultados semelhantes foram encontrados em outros estudos utilizando a hiperinsuflação com o ventilador mecânico. ${ }^{(15,16)}$

\section{CONCLUSÃO}

Com base nos presentes achados sugere-se que a técnica do aumento do volume corrente inspiratório e consequente hiperinsuflação pulmonar utilizando o ventilador mecânico, promoveu um aumento do pico de fluxo expiratório que pode au- xiliar o deslocamento de secreções, sem promover uma instabilidade clínica ao paciente.

\section{REFERÊNCIAS}

1. Guglielminotti J. et al. Bedside detection of retained tracheobronchial secretions in patients receiving mechanical ventilation: Is it time for tracheal suctioning? Chest. 2000;118(4):1095-9.

2. Volpe MS. Ventilation Patterns Influence Airway Secretion Movement. Respir Care. 2008;53(10):1287-94.

3. Levitzky MG. Fisiologia pulmonar. São Paulo: Manole; 2004.

4. Fuhr T, Jaeger RJ. The Role of the HeringBrever Deflationary Reflex in the Control of Spontaneous Human Respiration. Journal of Automatic Control. 2002;12(2):56-63.

5. Tryfon S. et al. Hering-Brever Reflex in Normal Adults and in Patients With Chronic Obstructive Pulmonary Disease and Interstitial Fibrosis. Respiration. 2001;68:140-144.

6. Avena KM. Efeitos da tosse manualmente assistida sobre a mecânica do sistema respiratório de pacientes em suporte ventilatório total. J Bras Pneumol. 2008;34(6):380-6.

7. Unoki T. Effects of expiratory rib Cage compression and/or prone position on oxygenation and ventilation in mechanically ventilated rabbits with induced atelectasis. Respir Care. 2004;48(8):896-901.

8. Lucangelo ES. et al. Respiratory mechanics derived from signals in the ventilador circuit. Respir Care. 2005;50(1):55-63.

9. Falta colocar

10. Pinheiro $\mathrm{CH}$. et al. Modificação do padrão respiratório melhora o controle cardiovascular na hipertensão essencial. Arqui Bras Cardiol. 2007;88(6):651-9.

11. Michard F. Changes in Arterial Pressure during Mechanical Ventilation. Anesthesiology. 2005;103(2):419-28. 
12. Singer M. et al. Hemodynamic effects of manual hyperinflation in critically ill mechanically ventilated patients. Chest. 1994;106:1182-7.

13. Miske JL. et al. Use of the mechanical In-Exsufflator in pediatric patients with neuromuscular disease and impaired cough. Chest. 2004;125(4):1406-12.

14. Winck J. C. et al. Effects of mechanical Insufflation-Exsufflation on respiratory parameters for patients with chronic airway secretion encumbrance. Chest. 2004;126:774-80.

15. Berney S, Denehy L. A comparison of the effects of manual and ventilator hyperinflation on static lung compliance and sputum production in intubated and ventilated intensive care patients. Physioter Res Int. 2002;7:100-8.

16. Savian C. et al. Comparison of the effectiveness of manual and ventilator hyperinflation at different levels of positive end-expiratory pressure in artificially ventilated and intubated intensive care patients. Heart Lung. 2006;35:334-41.

17. Guyton AC, Hall JE. Tratado de fisiologia médica. Rio de Janeiro: Elsevier; 2006.

18. West J. Fisiologia respiratória. São Paulo: Manole; $2 \mathrm{OO} 2$.

19. Irwin S, Tecklin J, Fisioterapia cardiopulmonar. São Paulo: Manole; 2003. 